\title{
Long Term Productivity and Collaboration in Information Science ${ }^{1}$
}

\author{
Jonathan M. Levitt and Mike Thelwall
}

Statistical Cybermetrics Research Group, School of Mathematics and Computing, University of Wolverhampton, Wulfruna Street, Wolverhampton WV1 1LY, UK.

Funding bodies have tended to encourage collaborative research because it is generally more highly cited than sole author research. But higher mean citation for collaborative articles does not imply collaborative researchers are in general more research productive. This article assesses the extent to which research productivity varies with the number of collaborative partners for long term researchers within three Web of Science (WoS) subject areas: Information Science \& Library Science (IS\&LS), Communication and Medical Informatics. When using the whole number counting system, researchers who worked in groups of 2 or 3 were generally the most productive, in terms of producing the most papers and citations. However, when using fractional counting, researchers who worked in groups of 1 or 2 were generally the most productive. The findings need to be interpreted cautiously, however, because authors that produce few academic articles within a field may publish in other fields or leave academia and contribute to society in other ways.

\section{Introduction}

Funding bodies take into account numerous factors when allocating research funding. For instance, they try to fund research that is likely to make a significant contribution to knowledge or have a substantial impact in other ways. Another factor is that for some research projects an interdisciplinary approach is particularly suitable. In this context, funding bodies are also interested in whether collaborative researchers are likely to be particularly productive, because some (such as the European Commission) sometimes include collaboration as a requirement for funding.

In this article the term 'collaboration' denotes 'co-authorship' even though the two are sometimes different (Katz \& Martin, 1997). Not all collaboration results in co-authorship (Gotzsche, Hróbjartsson, Johansen, Haahr, Altman, \& Chan, 2007); for example, experienced researchers who give feedback to doctoral students that they are not mentoring or supervising are unlikely to be listed as co-authors. Moreover, co-authors do not always make a substantial contribution to an article (Smith, 1994). Nevertheless, it seems reasonable to assume that in general co-authorship of a paper tends to indicate that a person has made a substantial contribution to it.

This article investigates the relationship between productivity of long-term researchers and the number of researchers within their collaborations, in order to assess the extent to which collaboration is bibliometrically advantageous when researchers are analysed over the longer term. On a large scale it is impossible to fully evaluate the productivity of an individual either

\footnotetext{
${ }^{1}$ Levitt, J. \& Thelwall, M. (in press). Long Term Productivity and Collaboration in Information Science. Scientometrics. doi: 10.1007/s11192-016-2061-8
} 
in general or in terms of their contribution to science. This article uses the term 'productivity' to denote the extent to which researchers contribute to scholarship through publishing articles and being cited in the Web of Science (WoS). Thus, scientific productivity is often narrowed down in bibliometric studies to either the amount of publications produced or the number of citations attracted. In the latter case, the rationale is that the number of citations received by a body of work tends to reflect how substantial its contribution is. The Discussion examines on a small-scale some of the ways, other than through authoring WoS articles, in which researchers contribute.

Although collaboration is a major goal of funding bodies, little research has been conducted on establishing the extent to which larger groups of researchers are more productive than smaller groups. Many studies have found that the mean citation level of groups of authors is generally higher than the mean citation level of articles by a sole author. But higher mean citation does not imply higher productivity; for example, a group of ten researchers who publish an article that is cited ten times are less productive, in terms of citations and number of articles, than ten researchers who each publish one article that is cited nine times.

\section{Background}

\section{1: Collaboration and citations}

Within science, many studies have shown that papers with more co-authors tend to attract more citations. For example, a study using the whole of WoS, which is dominated by the sciences, found a general trend for articles with more co-authors to have higher mean citation counts (Costas \& van Bochove, 2012). Similarly, a study of a sample of Italian articles, from a variety of areas of scholarship, found more highly authored articles to be both more highly cited and more highly rated by peer reviewers (Franceschet \& Costantini, 2010). At the level of individual scientific subjects, each additional author, to a maximum of 7 authors, increases the average number of citations per article in biomedical research, chemistry and mathematics (Glänzel, 2002). There is a correlation between collaboration and citation for Chinese molecular biology (Ma \& Guan, 2006), but not for well-known molecular biology research institutes (Herbertz, 1995).

Some studies of the social sciences, often using Social Science Citation Index (SSCI) data, have also found statistically significant associations between collaboration in general and citation, including for economics overall, but varied between countries (Levitt \& Thelwall, 2010) and for ecology overall, but not for co-authorships within the same institution (Leimu $\&$ Koricheva, 2005). From a slightly different perspective, the proportion of the most highly cited library and information science articles that are collaborative has increased over time but the same is not true for uncited articles (Levitt \& Thewall, 2009). Graduate students on a library and information science course also rated articles more highly if they had more than one author (Finlay, Ni, \& Sugimoto, 2012), suggesting that higher citation may reflect higher quality or utility within this area.

Not all investigations of the social sciences and humanities have found an association between collaboration level and an increased or decreased level of citation; these include investigations of physical education, recreation, and dance (Crase \& Rosato, 1992), finance (Avkiran, 1997), and two library science journals (Hart, 2007). 
Collaboration between researchers in different fields can lead to research that is more highly cited, although this depends upon the collaborating fields involved (Larivière \& Gingras, 2010; Levitt \& Thelwall, 2008). For example, collaboration between similar fields may be generating more citations than collaboration between distant fields (Yegros-Yegros, Rafols, \& D'Este, 2015).

\section{2: Citations and national or international collaboration}

Several studies of science have found that international articles by more than one author are particularly highly cited. For each of the 31 countries that published the largest number of science papers in 1995, internationally collaborative articles were more highly cited on average than non-international collaborative articles (Glänzel, 2000). Based upon SCI articles in 1998, the mean citation rate for SCI articles, excluding self-citations, increased at the same rate for national and international articles ( 0.5 citations for each author after the first author up to 12) although international collaborative articles received, on average, about 1 more citation, than did domestic collaborative articles (Persson, Glänzel \& Danell, 2004). Based on nearly half a million UK SCI publications from 1981 to 1994 (Katz \& Hicks, 1997), articles by authors from two countries received, on average, about $50 \%$ more citations than articles by authors from a single country. Positive associations between international collaboration and citation rates have also been found for Scandinavian science (Glänzel, 2000), Brazilian science (Leta \& Chaimovich, 2002), New Zealand science (Goldfinch, Dale, \& DeRouen, 2003), and Danish industry (Frederiksen, 2004). In contrast, papers with at least one Harvard University co-author attract less citations the greater the physical distance between the affiliations (Lee, Brownstein, Mills, \& Kohane, 2010), suggesting that international collaboration is ineffective for Harvard authors. This is probably a special case for one elite university and hence does not negate the other findings.

At the level of individual subjects, positive associations have been found between citations and international collaboration for Chilean physics (Vogel, 1997), HIV/AIDS in Nigeria (Uthman, 2008) and wood preservative chemical research (Yi, Ao, \& Ho, 2008). An investigation of Spanish Gastroenterology, Cardiovascular Systems and Neuroscience SCI articles published between 1991 and 1993 found that international collaboration helped Spanish researchers to publish in higher impact journals (Bordons, Gomez, Fernandez, Zulueta \& Mendez, 1996) and thus, presumably, to attract more citations. Similarly, an investigation of chemistry papers published in 1995 found that for 33 of the 36 countries, international collaborative papers were, on average, more highly cited than non-international collaborative papers (Glänzel \& Schubert, 2001). These findings demonstrate that - in contrast to international collaboration, national collaboration may not always be associated with higher impact research. An alternative explanation may be that more capable researchers are more able to establish international collaboration, or, that higher quality research projects are more likely to attract international teams.

One concern, when investigating collaboration, is that articles by multiple authors seem more likely to be self-cited as there are more authors to self-cite. In partial response, an investigation of astronomy research in the Netherlands (Van Raan, 1998) concluded that higher rates of self-citation in international collaboration do not play a significant role in increasing the citation impact of internationally collaborated articles. A similar result was found for the whole of WoS (Costas \& van Bochove, 2012). 
Within the social sciences, links have been found between higher citation levels and international collaboration for Brazilian management science (Pereira, Fischer, \& Escuder, 2000), library and information science (Levitt \& Thelwall, 2009) and economics (Levitt \& Thelwall, 2010).

A few studies of collaboration have compared more than one country. An investigation of 837 papers published in Oecologia between 1998 and 2000 found a citation advantage for collaborative articles that was higher for US authors than for European authors, although international collaborations were not more highly cited than national collaborations (Leimu \& Koricheva, 2005). Another study generalised this result by showing that the citation advantage gained through international collaboration varied by discipline and country, tending to be greatest in countries with the lowest citation impact and in social sciences and engineering (Lancho-Barrantes, Guerrero Bote, \& de Moya Anegón, 2013).

\section{3: Citation, productivity and group size}

Some research has also been conducted on the extent to which productivity depends on group size. A pilot study investigated the extent to which the citation impact of articles published in three sub-branches of physics in 2004 varied according to group size (Levitt, 2011). In these sub-branches, when using fractional counting system (that divides the credit for an article equally amongst the authors), the citation productivity was higher for smaller groups than for larger groups. In order to identify changes over time, the productivity of long-term researchers in the WoS category of Information Science \& Library Science (IS\&LS) was investigated; long-term researchers were defined as researchers who authored at least one IS\&LS article in both 1998-2002 and 2008-11. For 7 of the 9 years investigated, citation productivity, as measured by the fractional counting system, was highest for group-sizes between 2 and 3 (Levitt \& Thelwall, 2013).

Gazni and Didegah (2011), in an investigation of WoS articles with at least one author affiliated with Harvard University, found a significant positive correlation between the number of co-authors and the number of citations. Franceschet \& Costantini (2010), in an investigation of 970 Italian economics and statistics articles, found that the citation level increased as the number of authors increased from 1 to 2 and from 2 to 3 or more. Didegah and Thelwall (2013), in a study of nanotechnology articles, found that the number of authors was not a significant determinant of citations once other factors, such as various types of internationality, were taken into account. Levitt (2015), in an investigation of nineteen social science disciplines for the years 1995, 1998, 2001, 2004 and 2007, found that, although the mean citation counts rose as the number of authors increased from 1 to 4 , by far the largest rise occurred when the number of authors increased from 1 to 2 .

\section{Productivity and careers}

Studies that investigate academic productivity in terms of publication counts tend to assume that researchers that produce more publications are more productive but this is not necessarily true. To give an extreme example, a laboratory head might co-author hundreds of articles every year without contributing anything to them whereas a part-time $\mathrm{PhD}$ student might write a single article during their lifetime but be a highly productive member of society in their main job outside of academia. Alternatively, a person contributing one information science article during their lifetime could actively publish in other disciplines rather than being unproductive. Moreover, gender and disability can also affect productivity in ways that 
are not relevant to studies of productivity because women tend to take more leave for childcare (Wolfinger, Mason, \& Goulden, 2008) and some types of disability (e.g., see: HEFCE, 2011) make it more difficult to produce some types of research. It is therefore important to understand what types of people produce few articles in order to draw conclusions from any study of productivity in terms of publications produced within a field.

PhD students are a type of researcher that may produce a small number of articles and then leave academia. Perhaps half of all doctoral students do not complete their studies (Lovitts, 2001) and some of these are likely to produce publications before switching to a different career. Failure to complete a doctorate is not necessarily a career failure because the student may leave after realising that a $\mathrm{PhD}$ will not help them to get the job that they want (Golde, 1998). A survey of US doctorate recipients in 2000 found that just over half of those that were US citizens intended to work in higher education (Hoffer, Dugoni, Sanderson, Sederstrom, Ghadialy, \& Rocque, 2001). Assuming that not all of these successful, it is likely that under half of US doctorates become employed in a role in which producing scholarly publications would be relevant to their career productivity.

Industry also produces a substantial number of publications (e.g., Frederiksen, 2004) but since commercial organisations have profit as a primary goal it seems unlikely that many industrial scientists have authoring publications as their main objective. In contrast, although publications are important in some industries, such as pharmaceutics (Hirsch, 2009), a paper may often be produced as a side effect of commercial jobs rather than as the end result of a task. Thus, an apparently unproductive industrial researcher with a single publication might instead be a highly productive member of her organisation that has cemented her reputation within industry by producing an academic output. Alternatively, an academic researcher may produce relatively few articles because they have switched from a non-publishing commercial or government sector job (Dietz \& Bozeman, 2005).

\section{Research question}

This study investigates the relationship between long term productivity and collaborative group size for researchers who published in the related WoS categories of IS\&LS, Communication and Medical Informatics. These three similar subjects were chosen in order to identify fine-grained differences. This extends a previous investigation (Levitt \& Thelwall, 2013), through adding two additional categories, to shed light on the extent to which the findings on productivity for IS\&LS are discipline-dependant.

Although numerous studies have found that collaborative research is, in general, more highly cited than sole author research, no study has investigated collaboration and citation for long term researchers. This is important to assess, because (a) it enables evaluation of not only the mean citation of researchers to be assessed but also the number of articles they publish and (b) it prevents the findings being distorted by researchers who have published only one article and then subsequently left academia. In order to fill this gap for one particular subject area, this article addresses the following research question: To what extent does the research productivity of long-term IS\&LS, Communication and Medical Informatics researchers, when measured by whole and fractional article and citation counts, vary with their typical number of collaborative partners? 


\section{Methods and data}

This study investigates the extent to which the research productivity of long-term researchers varied with the average group size of the authors. Research productivity is measured by calculating the fractional article contribution and fractional citation contribution in the period assessed. Fractional contributions use the fractional counting system, recommended by Price (1981) and used in several investigations (e.g., Burrell \& Rousseau, 1995; Glänzel \& De Lange, 2002). In the fractional counting system the total credit for a sole author article is the same as for a collaborative article, but the authors of collaborative articles share equal fractions of the credit for their articles. The whole counting system, in which the credit for the article allocated to the author is irrespective of the number of authors, can be misleading because an article by ten co-authors receives ten times the total credit of an article by a single author. Other ways of being productive are excluded from the main analysis in order to give clear results and due to the lack of comparable data sources. These include publishing books, conference papers, research reports and patents as well as teaching, managing PhD students, research groups or projects, commercialising research, conducting science outreach activities and providing expert advice to the media, government or business.

This article investigates the productivity of long-term researchers for the period 1996 to 2009. A long-term researcher was defined as someone who authored at least one article in the period 1993 to 1996 and at least one article in the period 2009 to 2012. Qualifying researchers must therefore have been active in the field for a minimum of 13 years. 2009 is the cut-off point, in order to have a citation window of at least 4 years. The decision to not limit 'long-term' to researchers who published in the first and last year of the data analysed was taken to avoid restricting the sample to researchers who published in 1996 and 2009. This definition precluded the investigation of more recently published research than 2009 . Whilst it may be interesting to know about productivity after 2009, this was not investigated as it is not central to the research question and would have entailed extensive additional data collection and analysis. Our study investigates people who definitely were researchers in at the beginning and end of the period, whereas people who published in 2009, might have been retired from research in that year.

The interval was divided into two time-periods (1996 to 2002 and 2003 to 2009) in order to identify changes over time. A seven year period was examined in order to monitor research over a reasonably long duration. The time period was not divided into more than two periods, as the findings for the two periods did not vary much and using more periods would have reduced the sample sizes in each period.

This article uses four metrics of productivity that each indicates a different aspect of productivity. The whole metrics (whole article count and whole citation counts) depict productivity of the individual researchers. These metrics indicate the extent to the number of articles authored and citations accrued by a researcher vary according to the size of collaborative group.

The fractional metrics (fractional article count and fractional citation counts) depict productivity of aggregates of researchers. These metrics indicate the extent to the number of articles authored and citations accrued by aggregates of researchers vary according to the size of collaborative group. For each metric a 95\% confidence interval for each data point was calculated using bootstrapping. 
The mean number of citations per article was not used as an indicator of productivity, because it can be misleading. For instance, consider the following scenarios: Group A produced 1 article which received 10 citations in the same time that Group B produced 10 articles that received 90 citations. Although Group A has a higher mean citation it was less productive. The productivity of aggregates of researchers reflects the output of a hypothetical 1000 typical researchers in each size category, and seems is a strong indicator of productivity of funded research. For the three WoS categories, bibliographic data was obtained from the Social Sciences Citation Index in 2014, and for each time period the names of researchers who published in the period were extracted. The mean group size (called here 'collaboration level') of the researcher was obtained by adding the number of authors in the category and period authored by the researcher and dividing by the number of articles published by the researcher in the period (Table 1). Authors were identified on the basis of their surnames and initials. As the author names were restricted to a single WoS category there the cases of different authors with the same surname and initials seem unlikely to systematically affect the results.

Table 1: The number of authors (i.e., sample size) at each level of collaboration. For example, 3 indicates people for which the average number of authors on their articles is at least 3 but less than 4.

\begin{tabular}{|l|l|l|l|l|l|l|l|l|}
\hline $\begin{array}{l}\text { Collaboration level } \\
\text { Sample }\end{array}$ & 1 & 2 & 3 & 4 & 5 & 6 & 7 & 8 \\
\hline LIS 1996-2002 & 3,773 & 4,124 & 2,911 & 1,790 & 960 & 607 & 352 & 278 \\
\hline LIS 2003-09 & 7,827 & 9,528 & 7,372 & 4,191 & 2,348 & 1,623 & 933 & 594 \\
\hline Com 1996-2002 & 2,456 & 2,734 & 1,902 & 916 & 581 & 318 & 198 & 155 \\
\hline Com 2003-09 & 3,837 & 6,112 & 5,496 & 3,109 & 1,502 & 1,123 & 677 & 345 \\
\hline MI 1996-2002 & 789 & 2,403 & 3,533 & 3,550 & 2,918 & 1,783 & 1,047 & 721 \\
\hline MI 2003-09 & 593 & 2,452 & 4,342 & 5,126 & 4,328 & 3,412 & 2,204 & 1,452 \\
\hline
\end{tabular}

\section{Findings}

The findings on productivity are presented in Figures 1 to 4 . Productivity is measured in Figure 1 by whole article count, in Figure 2 by whole citation count, in Figure 3 by fractional article count, in Figure 4 by fractional citation count. In each figure the graphs indicate the mean productivity of the authors in the levels of authorship and the error bars indicate $95 \%$ confidence intervals for the means. These tend to overlap for lines that are close together in the graphs, indicating that the differences between close lines are not statistically significant. The confidence intervals between lines that are not close together tend not to overlap, however, suggesting that these are statistically significantly different. The lines labelled 'LIS 1996-2002', 'Com 1996-2002' and 'MI 1996-2002' denote the productivity of IS\&LS, Communication and Medical Informatics authors in 1996-2002 and 'LIS 2003-09', 'Com 2003-09' and 'MI 2003-09' the productivity of IS\&LS, Communication and Medical Informatics authors in 2003-09. 


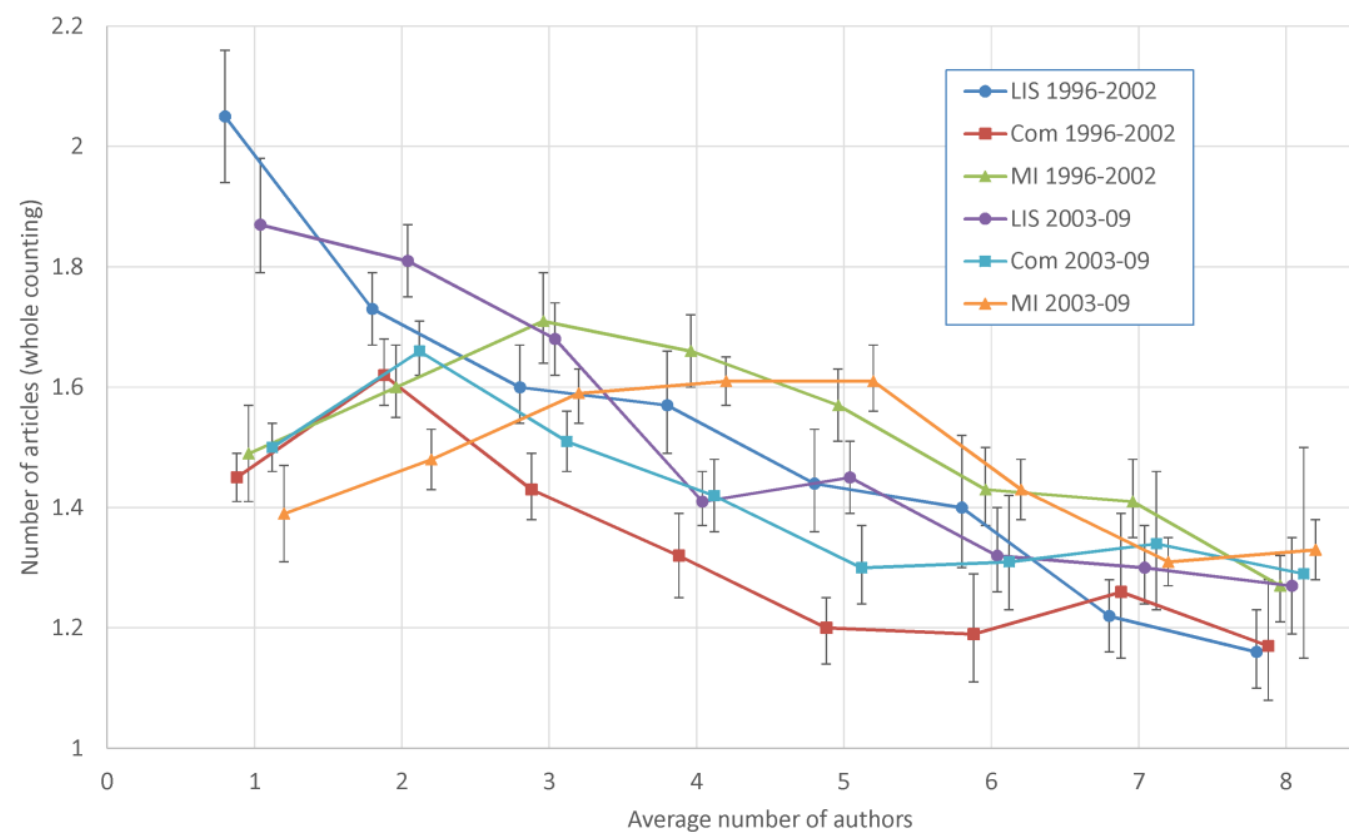

Figure 1: Average number of articles produced per author by collaboration level (whole article counting), measured separately for three subjects and two date ranges (six combinations) for long term researchers. Jitter has been added to the author numbers for the graph to prevent overlaps between the error bars.

In Figure 1, for both periods the collaborative level with the highest whole article count was 1 for LIS authors, 2 for Com authors and 3 or 4 for MI authors. For both periods, the collaborative level with the lowest whole article count was 8 for LIS authors, 7 or 8 for Com authors and 7 or 8 for MI authors. For both periods the range of whole article count was the highest for LIS. Amongst all the subjects and periods the highest whole article count was 2.2.

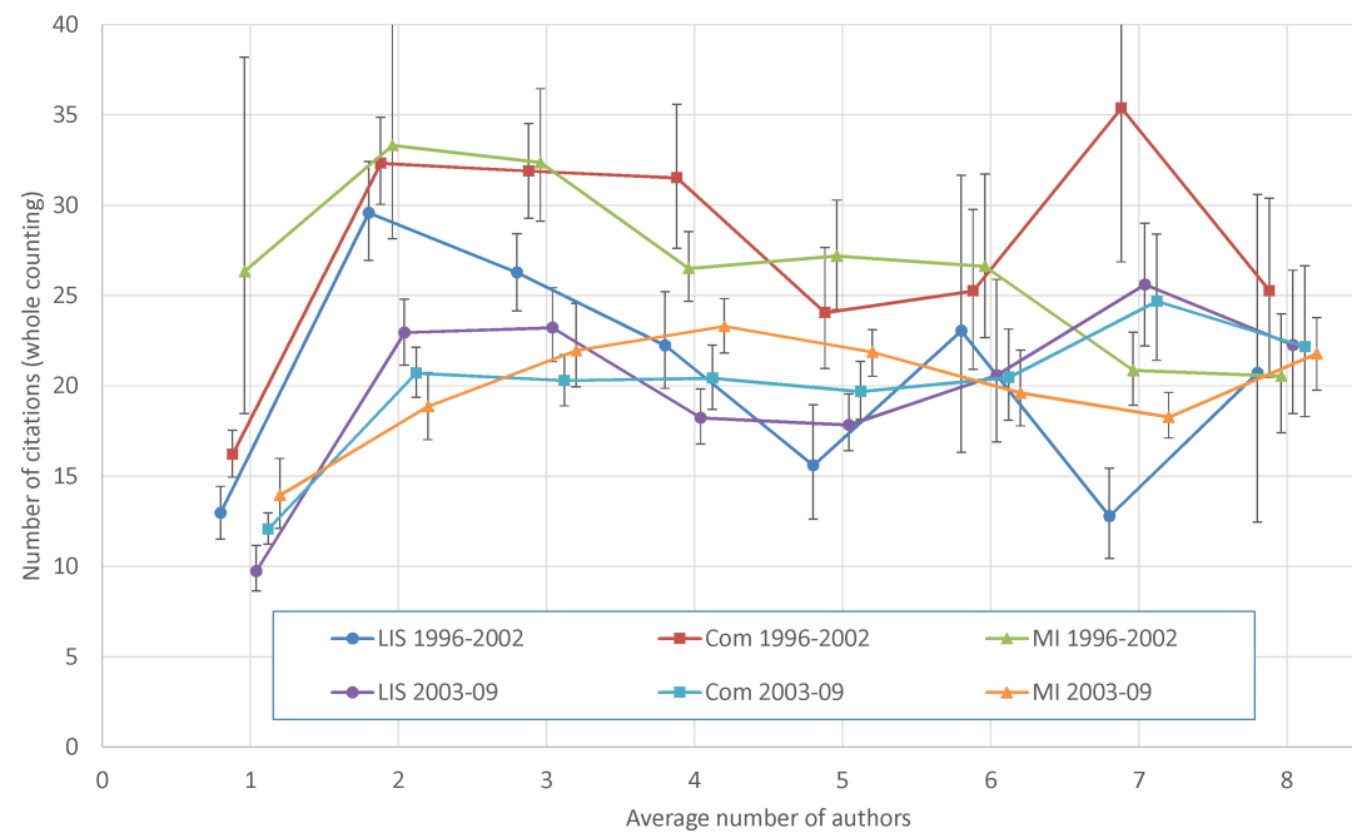

Figure 2: Average number of citations produced per author by collaboration level (whole citation counting), measured separately for three subjects and two date ranges for long term researchers. Jitter has been added to the author numbers for the graph to prevent overlaps between the error bars. 
The findings for citation counts are different from the findings for article counts. In Figure 2, for both periods and all subjects, authors with a collaborative level of 1 had a substantially lower mean whole citation count than authors with a collaborative level of 2 . Taken across subjects and periods, the lowest mean citation counts were 14.8 and 21.7 for the collaborative levels of 1 and 5, and highest mean citation counts were 27.4 and 27.1 for the collaborative levels of 2 and 3 . For both periods the range of whole citation count was the highest for LIS.

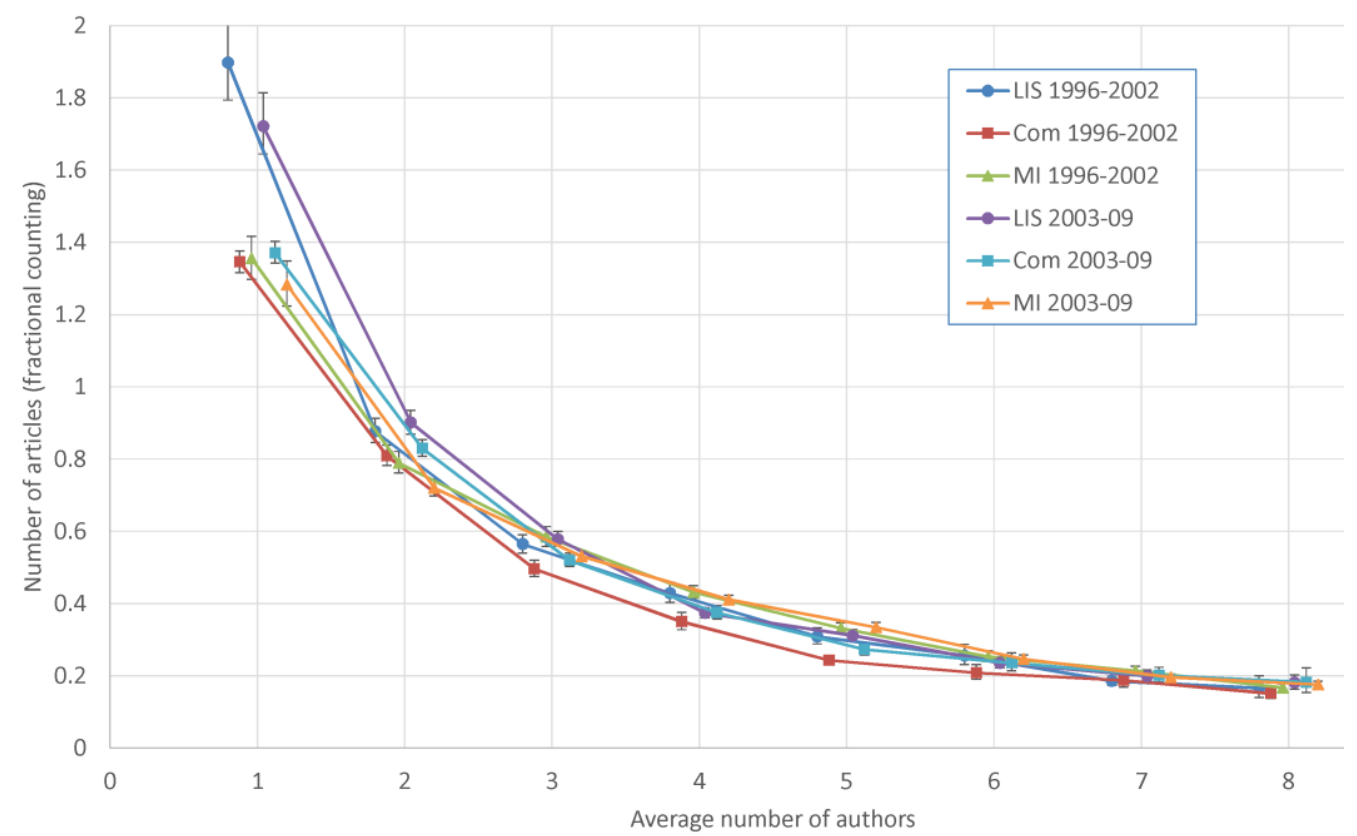

Figure 3: Average number of articles produced per author by collaboration level (fractional article counting), measured separately for three subjects and two date ranges for long term researchers. Jitter has been added to the author numbers for the graph to prevent overlaps between the error bars.

In Figure 3, for both periods and for all subjects, authors with a collaborative level of 1 had the highest fractional article count; the count declined steadily with increased collaborative level. 


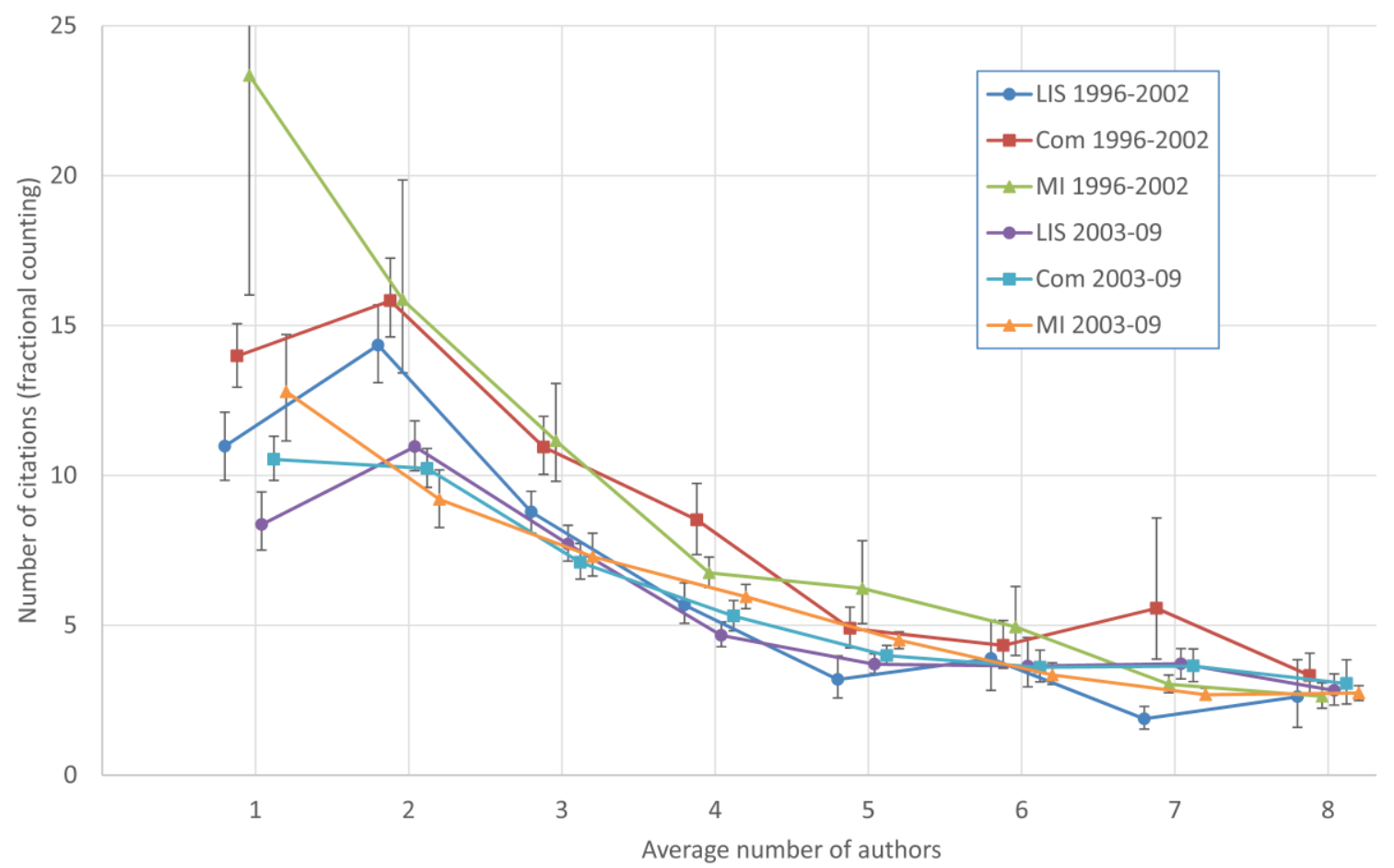

Figure 4: Average number of citations produced per author by collaboration level (fractional citation counting), measured separately for three subjects and two date ranges for long term researchers. Jitter has been added to the author numbers for the graph to prevent overlaps between the error bars.

In Figure 4, for both periods and all subjects, authors with a collaborative level of 1 or 2 had the highest fractional citation count; the count declined steadily when the collaborative level increased from 2 to 5 .

\section{Discussion}

For all three categories individual researcher productivity, when measured by whole article counts, tended to decrease with increased co-author group size. However, apart from IS\&LS this decrease was not substantial. Perhaps this is in part due to the multi-faceted nature of that subject sometimes leading to members of larger teams making relatively peripheral contributions. For all three categories, individual productivity, when measured by whole citation counts, tended to increase as the group size went from 1 to 2 , and thereafter tended to undulate. This indicates that long term researchers typically working in group sizes of 2 tended to have the highest individual citation productivity.

For all categories aggregate productivity, as measured by fractional article counting, decreased rapidly with increased co-author group size. For all categories aggregate productivity, as measured by fractional citation counting, was highest for group sizes of 1 or 2. Thus long term researchers typically working in group sizes of 1 or 2 tended to have the highest aggregate citation productivity.

The findings on whole metrics for long term researchers are consistent with the findings of numerous studies for all researchers that have found that citation of articles increases as the number of authors increases (e.g., Herbertz, 1995; Glänzel, 2002; Leimu \& Koricheva, 2005; Ma \& Guan, 2006; Franceschet \& Costantini, 2010; Levitt \& Thelwall, 2010; Costas \& van Bochove, 2012). This similarity in findings between long term and all researchers tends to 
suggest that the relationships between productivity and collaboration that apply to long tern researchers could also apply to short-term researchers and, in particular, that our findings may not be substantially different if short term researchers were included in the data. However whole number counts do not reflect the same traits as mean citation counts per article; the former gauges the productivity of individual researchers whereas the latter gauges the strength of articles. The fractional counting results are therefore more useful.

The findings may depend on the levels of interdisciplinarity of the subject categories investigated because researchers within interdisciplinary areas may be active for a long time in multiple areas but only publish occasionally in each one, hence appearing to be less productive. In particular, it could be expected that large groups tend to be the most interdisciplinary. Researchers who tend to work in large groups would therefore appear to be unproductive in any given field because these are the most likely to not be core members of the field analysed. For the three subject categories and both of the periods 1996 to 2002 and 2003 to 2009, data was collected on WoS categories that overlapped substantially with any of the categories. Table 2 presents the percentages for all categories in which the overlap, measured as a percentage of all articles in the category, exceeded 5\% for at least one of the periods.

Table $2 \mathrm{WoS}$ categories that overlapped with the three categories associated with information science.

\begin{tabular}{|l|r|r|}
\hline \multicolumn{1}{|c|}{ IS\&LS } & $\mathbf{1 9 9 6}$ to 2002 & $\mathbf{2 0 0 3}$ to 2009 \\
\hline Computer science information systems & $37.3 \%$ & $32.9 \%$ \\
\hline Computer science interdisciplinary applications & $13.0 \%$ & $9.5 \%$ \\
\hline Management & $6.1 \%$ & $8.5 \%$ \\
\hline Multidisciplinary sciences & $9.2 \%$ & $5.8 \%$ \\
\hline Communication & $4.0 \%$ & $5.3 \%$ \\
\hline Medical informatics & $7.6 \%$ & $3.0 \%$ \\
\hline \multicolumn{1}{|c|}{ Communication } & $\mathbf{1 9 9 6}$ to 2002 & $\mathbf{2 0 0 3}$ to 2009 \\
\hline Business & $10.0 \%$ & $10.3 \%$ \\
\hline IS\&LS & $8.8 \%$ & $8.8 \%$ \\
\hline Linguistics & $6.5 \%$ & $8.3 \%$ \\
\hline Psychology social & $10.1 \%$ & $7.4 \%$ \\
\hline Psychology applied & $3.5 \%$ & $7.2 \%$ \\
\hline Political science & $8.4 \%$ & $5.9 \%$ \\
\hline Film radio television & $4.1 \%$ & $5.7 \%$ \\
\hline Sociology & $5.2 \%$ & $5.1 \%$ \\
\hline & $\mathbf{1 9 9 6}$ to $\mathbf{2 0 0 2}$ & $\mathbf{2 0 0 3}$ to 2009 \\
\hline Health care sciences services & $25.0 \%$ & $35.4 \%$ \\
\hline Mathematical computational biology & $28.6 \%$ & $33.5 \%$ \\
\hline Computer science interdisciplinary applications & $39.4 \%$ & $32.8 \%$ \\
\hline Engineering biomedical & $31.1 \%$ & $26.0 \%$ \\
\hline Computer science information systems & $28.8 \%$ & $24.9 \%$ \\
\hline Public environmental occupational health & $23.0 \%$ & $23.8 \%$ \\
\hline Statistics probability & $18.4 \%$ & $20.6 \%$ \\
\hline Medicine research experimental & $18.1 \%$ & $19.0 \%$ \\
\hline Computer science theory methods & $8.2 \%$ & $8.1 \%$ \\
\hline Medicine general internal & $1.9 \%$ & $6.1 \%$ \\
\hline IS\&LS & $14.5 \%$ & $5.3 \%$ \\
\hline
\end{tabular}


Table 2 indicates substantial differences between the three subject categories. Although the percentages of overlap in general varied little between the two periods, the percentage overlap of IS\&LS with Medical informatics for 1996 to 2002 was more than double the percentage for 2003 to 2009 and the percentage overlap of Medical informatics with Medicine general internal for 1996 to 2002 was less than a third of the percentage for 2003 to 2009. There were substantial levels of overlap between IS\&LS and Communication and IS\&LS and Medical informatics. However, only two categories overlapped substantially with more than one of the subjects (Computer science information systems and Computer science interdisciplinary applications both overlapped substantially with IS\&LS and Medical informatics).

In the table IS\&LS overlapped by over $5 \%$ with 6 categories, Communication with 8 categories and Medical informatics with 11 categories. The overlap for IS\&LS exceeded $15 \%$ for only 1 category and for Communication did not exceed $15 \%$ for any of the categories; in contrast the level of overlap for Medical informatics exceeded $15 \%$ for as many as 8 categories. The categories that overlapped strongly with Medical informatics included not only medical categories (e.g., Medicine research experimental), but also mathematical (Statistics probability) and computer science (Computer science information systems) categories. Further evidence for the substantially higher level of Multidisciplinarity of Medical informatics is that the percentages of articles in more than one category for 1996 to 2002 was $60.7 \%$ for IS\&LS, $65.5 \%$ for Communication and $100 \%$ for Medical informatics, and for 2003 to 2009 it was $55.8 \%$ for IS\&LS, $64.3 \%$ for Communication and $98.5 \%$ for Medical informatics. A possible reason why the much higher levels of multidisciplinarity of Medical informatics are not reflected in Figures 1 to 4 is that researchers in large group sizes might not be not disproportionately unproductive within the discipline..

All scientometric investigations of productivity that use the number of articles produced by a researcher or research group as the sole indicator of productivity are oversimplifications because researchers can be productive in other ways. This is a major limitation of the current study because it may be that the group sizes that are apparently the least productive instead contain researchers that are the most productive in other ways. For example, $\mathrm{PhD}$ students may disproportionately author alone or with their main supervisor, and may disproportionately leave academia to pursue non-academic jobs. A small scale investigation of 100 first-named authors of an IS\&LS article in 1996 who did not publish any other IS\&LS articles in the period 1993 to 2012 (see online supplement: https://dl.dropboxusercontent.com/u/14001543/ISLSAuthorCareers.docx) confirmed that many had left academia to pursue other careers although some had also contributed to other disciplines instead.

\section{Limitations and Conclusions}

An important limitation is that this study was restricted to three subject categories. It seems likely that some of the results do not apply to categories in general. This applies particularly to the findings on the whole counts that vary more between categories of group size than the findings for the fractional counts. But as the findings are very similar for subject categories with widely differing levels of multidisciplinarity, they may apply to a substantial proportion of categories. The similarity in the findings of each subject category between productivity in 1996 to 2002 and 2002 and 2009, indicates that the findings are unlikely to be much different if the periods investigated were altered by a few years (e.g., 1999 to 2005 and 2006 to 2012). 
As mentioned in the Background, several studies of science have found that international articles by more than one author are particularly highly cited. In principle part of the higher productivity of groups of authors could be from international collaboration. Whilst it may be interesting to assess the extent to which the findings differ between international and noninternational collaboration this is outside the scope of this investigation.

This investigation found that a substantial number of researchers after publishing one Web of Science information science article either conducted research outside information science or stopped conducting research. Some of these appear to have been productive in other ways than academic authoring, undermining the validity of the results to some extent. Future studies may wish to find alternative ways to assess the long term productivity of groups of researchers.

The collaborative level with the highest level of whole article output was subject dependant (despite the similarity between the subjects), in that it was 1 for LIS, 2 for Communication and 3 or 4 for Medical Informatics. However, many of the findings applied to all three subjects; for instance, authors with a collaborative level of 1 had the highest fractional article count, and authors with a collaborative level of 1 or 2 had the highest fractional citation count.

Overall, the group size that on average published most articles (fractional counting) was for all three subjects 1 , and the group size that on average had most citations (fractional counting) varied between the three subjects from 1 to 2 . Thus, if funders wish to produce as many papers in total as possible in any of these three subject areas, the findings suggest that they should emphasise solo research, whereas if they are interested in total overall citation impact then they should fund solo research or pairs of researchers. This may not be the case in other areas (such as physical science) but future research is needed to assess this. Nonetheless, it raises the prospect that collaboration in research is not universally the most productive strategy.

This article contributes to methodology in that it uses metrics for gauging productivity at the individual and aggregate levels. The strength of collaborative research can be gauged roughly through citations at three levels: the article level (mean citation per article), at the individual level (citations per researcher) and at the aggregate level (fractional citations per researcher). Citation at the article level is a guide to the strength of an article, citation at the individual level a guide to the research contribution of individual researchers and citation at the aggregate level a guide to the strength of groups of researchers. We suggest that citation at the aggregate level is particularly suited for gauging the bibliometric value of funded research.

The case for funding collaborative research has been supported by studies of article strength, which found a higher mean citation in collaborative research. We contend that the aggregate productivity (gauged by the fractional article and citation counts) is more suited to comparing the productivity of funded researchers than article strength. This study found that both of these counts decreased as the size of group increased for longer term researchers. This suggests that collaboration does not aid overall productivity for long term researchers, as reflected in citation counts or numbers of articles. Moreover, researchers producing few papers in one field may also publish in other fields and may make substantial contributions to 
society in other ways and so high authorship productivity is not necessarily a relevant indicator.

\section{Acknowledgement:}

We wish to thank the first author's mother, Gertrude Levitt, for her very careful proofresiding and Mahshid Abdoli for her painstaking help with the data collection.

\section{References}

Avkiran, N.K. (1997). Scientific collaboration in finance does not lead to better quality research. Scientometrics, 39(2), 173-184.

Bordons, M., Gomez, I., Fernandez, M.T., Zulueta, M.A., \& Mendez, A. (1996). Local, domestic and international scientific collaboration in biomedical research. Scientometrics, 37(2), 279-295.

Costas, R. \& van Bochove, C. (2012). On the Relationship between Author Collaboration and Impact of Scientific Publications. Proceedings of 17th International Conference on Science and Technology Indicators, Montréal: Science-Metrix and OST, 447-448.

Crase, D. \& Rosato, F.D. (1992). Single versus multiple authorship in professional journals. Journal of Physical Education, Recreation and Dancing, 63(7), 28-31.

Didegah, F. \& Thelwall, M. (2013). Determinants of research citation impact in nanoscience and nanotechnology. Journal of the American Society for Information Science and Technology, 64(5), 1055-1064.

Dietz, J. S., \& Bozeman, B. (2005). Academic careers, patents, and productivity: industry experience as scientific and technical human capital. Research policy, 34(3), 349-367.

Finlay, S.C., Ni, C., \& Sugimoto, C.R. (2012). New methods for an old debate: Utilizing reader response to investigate the relationship between collaboration and quality in academic journal articles. Library \& Information Science Research, 34(2), 131-137.

Franceschet, M. \& Costantini, A. (2010). The effect of scholar collaboration on impact and quality of academic papers. Journal of Informetrics, 4(4), 540-553.

Frederiksen, L.F. (2004). Disciplinary determinants of bibliometric impact in Danish industrial research: Collaboration and visibility. Scientometrics, 61(2), 253-270.

Gazni, A. \& Didegah, F. (2011). Investigating different types of research collaboration and citation impact: a case study of Harvard University's publications. Scientometrics 87(2), 251-265.

Glänzel, W. (2000). Science in Scandinavia: A bibliometric approach. Scientometrics, 48(2), 121-150.

Glänzel, W. (2002). Coauthorship Patterns and Trends in the Sciences (1980-1998): A Bibliometric Study with Implications for Database Indexing and Search Strategies. Library Trends, 50(3), 461-473.

Glanzel, W., \& Schubert, A. (2001). Double effort = double impact? a critical view at international co-authorship in chemistry. Scientometrics, 50(2), 199-214.

Golde, C. M. (1998). Beginning graduate school: Explaining first-year doctoral attrition. New directions for higher education, 1998(101), 55-64.

Goldfinch, S., Dale, T., \& DeRouen, K. (2003). Science from the periphery: Collaboration, networks and 'periphery effects' in the citation of New Zealand crown research institutes articles, 1995-2000. Scientometrics, 57(3), 321-337.

Gotzsche, P. C., Hróbjartsson, A., Johansen, H. K., Haahr, M. T., Altman, D. G., \& Chan, A. (2007). Ghost authorship in industry-initiated randomised trials. PLoS Medicine, 4(1), 0040019. DOI: 10.1371/journal.pmed.0040019 
Hart, R.L. (2007). Collaboration and article quality in the literature of academic librarianship. Journal of Academic Librarianship, 33(2), 190-195.

HEFCE (2011). Assessment framework and guidance on submissions. http://www.ref.ac.uk/pubs/2011-02/

Herbertz, H. (1995). Does it pay to cooperate - a bibliometric case-study in MolecularBiology. Scientometrics 33(1), 1171995.

Hirsch, L. J. (2009). Conflicts of interest, authorship, and disclosures in industry-related scientific publications: the tort bar and editorial oversight of medical journals. In Mayo Clinic Proceedings 84(9), 811-821.

Hoffer, T. B., Dugoni, B. L., Sanderson, A. R., Sederstrom, S., Ghadialy, R., \& Rocque, P. (2001). Doctorate Recipients from United States Universities: Summary Report 2000. Survey of Earned Doctorates. http://files.eric.ed.gov/fulltext/ED459639.pdf

Katz, J.S. \& Hicks, D. (1997). How much is a collaboration worth? A calibrated bibliometric model. Scientometrics, 40(3), 541-554.

Katz, J. S., \& Martin, B. R. (1997). What is research collaboration? Research Policy, 26(1), $1-18$.

Lancho-Barrantes, B.S., Guerrero Bote, V. P., \& de Moya Anegón, F. (2013). Citation increments between collaborating countries. Scientometrics, 94(3), 817-831.

Larivière, V., \& Gingras, Y. (2010). On the relationship between interdisciplinarity and scientific impact. Journal of the American Society for Information Science and Technology, 61(1), 126-131.

Lee, K., Brownstein, J., Mills, R., \& Kohane, I., (2010). Does collocation inform the impact of collaboration? PLoS ONE, 5(12), e14279.

Leimu, R. \& Koricheva, J. (2005). Does scientific collaboration increase the impact of ecological articles? Bioscience, 55(438), 438-443.

Leta, J., \& Chaimovich, H. (2002). Recognition and international collaboration: The Brazilian case. Scientometrics, 53(3), 325-335.

Levitt, J.M., \& Thelwall, M. (2008). Is multidisciplinary research more highly cited? A macrolevel study. Journal of the American Society for Information Science and Technology, 59(12), 1973-1984.

Levitt, J.M., \& Thelwall, M. (2009). Citation levels and collaboration within library and information science. Journal of the American Society for Information Science and Technology, 60(3), 434-442.

Levitt, J.M., \& Thelwall, M. (2010). Does the higher citation of collaborative research differ from region to region? A case study of Economics. Scientometrics, 85(1), 171-183

Levitt, J.M., \& Thelwall, M. (2013). The relationship between collaboration and productivity for long-term information science researchers. In: J. Gorraiz, E. Schiebel, C. Gumpenberger, M. Hörlesberger \& H. Moed (Eds.) 14th International Society of Scientometrics and Informetrics Conference - Proceedings. AIT Austrian Institute of Technology, Vienna, 1461-1468.

Levitt, J.M. (2011). Preliminary findings on whether it is good value for money to fund larger research groups. ISSI Newsletter, 7(3), 57-62.

Levitt, J.M. (2015). What is the optimal number of researchers for social science research? Scientometrics, 102(1), 213-225.

Lovitts, B. E. (2001). Leaving the ivory tower: The causes and consequences of departure from doctoral study. Lanham, MD: Rowman \& Littlefield.

Ma, N. \& Guan, J.C. (2005). An exploratory study on collaboration profiles of Chinese publications in Molecular Biology. Scientometrics, 65(3), 343-355. 
Pereira, J. C. R., Fischer, A. L., \& Escuder, M. M. L. (2000). Driving factors of high performance in Brazilian management sciences for the 1981-1995 period. Scientometrics, 49(2), 307-319.

Persson, O., Glanzel, W., \& Danell, R. (2004). Inflationary bibliometric values: The role of scientific collaboration and the need for relative indicators in evaluative studies. Scientometrics, 60(3), 421-432.

Smith, J. (1994). Gift authorship: a poisoned chalice? BMJ: British Medical Journal, 309(6967), 1456.

Uthman, O.A. (2008). HIV/AIDS in Nigeria: A bibliometric analysis. BMC Infectious Diseases, 8(2) 19.

Van Raan, A. F. J. (1998). The influence of international collaboration on the impact of research results. Some simple mathematical considerations concerning the role of selfcitations. Scientometrics, 42(3), 423-428.

Vogel, E.E. (1997). Impact factor and international collaboration in Chilean physics: 19871994. Scientometrics, 38(2), 253-263.

Wolfinger, N. H., Mason, M. A., \& Goulden, M. (2008). Problems in the pipeline: Gender, marriage, and fertility in the ivory tower. The Journal of Higher Education, 79(4), 388405.

Yegros-Yegros, A., Rafols, I., \& D’Este, P. (2015). Does interdisciplinary research lead to higher citation impact? The different effect of proximal and distal interdisciplinarity. PloS one, 10(8), e0135095.

Yi, H., Ao, X.L., \& Ho, Y.S. (2008). Use of citation per publication as an indicator to evaluate pentachlorophenol research. Scientometrics, 75(1), 67-80. 